Commun. Fac Sci. Univ. Ank. Ser. A1 Math. Stat.

Volume 69, Number 1, Pages 413-430 (2020)

DOI: $10.31801 /$ cfsuasmas. 666357

ISSN 1303-5991 E-ISSN 2618-6470

http://communications.science.ankara.edu.tr/index.php?series=A1

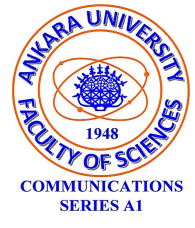

\title{
EQUIVALENCE CONDITIONS OF TWO SYSTEMS OF VECTORS IN THE TAXICAB PLANE AND ITS APPLICATIONS TO TAXICAB POLYGONS
}

\author{
İDRIS ÖREN AND HÜSNÜ ANIL ÇOBAN
}

\begin{abstract}
This study presents the conditions of $M_{T}(2)$-equivalence for two systems of vectors $\left\{x_{1}, x_{2}, x_{3}\right\}$ and $\left\{y_{1}, y_{2}, y_{3}\right\}$ in $R_{T}^{2}$, where $M_{T}(2)$ is the group of all isometries of the 2-dimensional taxicab space $R_{T}^{2}$. Firstly a minimal complete system of $M_{T}(2)$-invariants of $\left\{x_{1}, x_{2}, x_{3}\right\}$ is obtained. Then, using the conditions of $M_{T}(2)$-equivalence, an answer is given to the open problem posed in [10 p.428]. Furthermore, an algorithm is given for constructing taxicab regular polygons in terms of $M_{T}(2)$-invariants. This algorithm is general and useful to construct the taxicab regular $2 n$-gons and gives a tool to solve special cases of the open problem posed in 2 p.32]. Besides, both the conditions of the taxicab regularity of Euclidean regular polygons and Euclidean regularity of taxicab regular polygons are given in terms of $M_{T}(2)$-invariants.
\end{abstract}

\section{INTRODUCTION}

Many problems in applied algebra have symmetries or are invariant under certain natural transformations. In particular, all geometric magnitudes and properties are invariant with respect to the underlying transformation group. Properties in Euclidean geometry are invariant under the Euclidean group of rotations, reflections and translations; properties in projective geometry are invariant under the projective transformations, etc. This identification of geometry and invariant theory is expressed in Felix Klein's Erlanger Program (see detailed information in 14, p.14, 193]).

Let $R$ be the field of real numbers. Then the 2-dimensional taxicab space can be introduced by using the metric $d_{T}(x, y)=\left|x_{1}-y_{1}\right|+\left|x_{2}-y_{2}\right|$ instead of the well known Euclidean metric $d_{E}(x, y)=\sqrt{\left(x_{1}-y_{1}\right)^{2}+\left(x_{2}-y_{2}\right)^{2}}$, where $x=\left(x_{1}, x_{2}\right), y=\left(y_{1}, y_{2}\right) \in R^{2}$. This space will be denoted by $R_{T}^{2}$ which is known as taxicab plane geometry.

Received by the editors: October 10, 2017; Accepted: December 23, 2019.

2010 Mathematics Subject Classification. Primary 13A50, 51K05; Secondary 51M04,51K99.

Key words and phrases. Invariant, regular polygon, taxicab geometry.

(C)2020 Ankara University Communications Faculty of Sciences University of Ankara-Series A1 Mathematics and Statistics 
The taxicab metric $d_{T}(x, y)=\sqrt{p(x-y, x-y)+2\left|\left(x_{1}-y_{1}\right)\left(x_{2}-y_{2}\right)\right|}$ is also defined in the paper [3, Definition 3.1, p.302], where $p(x-y, x-y)$ is Euclidean inner product.

Let $M_{T}(2)=\left\{F: R_{T}^{2} \rightarrow R_{T}^{2}: F x=g x+b, \forall g \in D_{4}, b \in R_{T}^{2}\right\}$ which is known as taxicab group (see [8, [10, p.424]) be the group of all isometries of $R_{T}^{2}$, where the dihedral group $D_{4}$ is the (Euclidean) symmetry group of the square.

Let $M_{E}(2)=\left\{F: R^{2} \rightarrow R^{2}: F x=g x+b, \forall g \in O(2), b \in R^{2}\right\}$ which is known Euclidean motion group (see [10, p.424]) be the group of all isometries of the 2dimensional Euclidean space $R^{2}$ where the group $O(2)$ is the orthogonal group.

The complete system of $M_{E}(n)$-invariants of a system of the vectors $\left\{x_{1}, x_{2}, \ldots, x_{m}\right\}$ in the $n$-dimensional Euclidean space is given in [9, Theorem 6] and the complete system of relations between elements of this complete system is given in [9, Theorem 3] where $M_{E}(n)$ is an $n$-dimensional Euclidean motion group.

An aim of this study is to present the equivalence conditions of two systems of vectors $\left\{x_{1}, x_{2}, x_{3}\right\},\left\{y_{1}, y_{2}, y_{3}\right\}$ and to give a minimal complete system of $M_{T}(2)$ invariants of the vectors $\left\{x_{1}, x_{2}, x_{3}\right\}$ for taxicab plane geometry.

The taxicab geometry play an important role in ecology, optic, fire-spread simulation with square-cell, grid-based maps and nonlinear differential equations. Applications of the taxicab metric in ecology are also well-known. Ecologist have found taxicab metric $d_{T}$ a useful metric in the measurement of 'niche overlap' and notion of ecological distance between species.(see in papers [13, [11, [16], [4, [12], [7, [1]).

Let us give the well known theorem in the Euclidean geometry as; "If systems $\left\{x_{1}, x_{2}, \ldots, x_{m}\right\}$ and $\left\{y_{1}, y_{2}, \ldots, y_{m}\right\}$ of vectors in $R^{n}$ such that $d_{E}\left(x_{i}, x_{j}\right)=$ $d_{E}\left(y_{i}, y_{j}\right)$ for all $i, j=1,2, \ldots, m ; i \neq j$, then there exists a unique isometry $F$ of $R^{n}$ for which $F x_{i}=y_{i}$ for all $i=1,2, \ldots, m . "$.

The group of isometries of the taxicab geometry described, and the following open problem is given in [10]: "What (if any) is the taxicab metric analogue of the theorem above for Euclidean isometries?" In this study, an answer is given to this open problem.

Therefore, the following question is one of the fundamental problems of invariant theory (see [14, pp.15]).

"Given a geometric property $P$, find the corresponding invariants and vice versa. Is there an algorithm for this transition between geometry and algebra?"

Let $P$ be any taxicab regular polygon in the taxicab plane. For $P$, the following problems are important:

(1) The existence or non-existence of $P$.

(2) Which Euclidean regular polygons are also the taxicab regular, and which are not?

(3) Find an algorithm to construct taxicab regular polygons.

The above problems for $P$ are geometrically discussed in [2]. Regular polygons in the taxicab plane were studied by means of taxicab circles also in [6. Some 
regular polygons in the taxicab 3 -space are described in [15]. In papers [2, 6, the following corollaries are obtained:

(i) In 2, the existence of taxicab regular $2 n$-gons by means of taxicab circles is proved. Besides, the non-existence of taxicab regular triangle are proved geometrically, and the question "Does there exist any taxicab regular $(2 n-1)$-gons?" is posed as an open problem. In [6], the non-existence of taxicab regular triangles and pentagons are proved geometrically by means of taxicab circles.

(ii) In the papers 2, 6, it is proved that all Euclidean squares and some special Euclidean regular octagons are also taxicab regular, and vice versa.

(iii) To construct taxicab regular $2 n$-gons, a procedure is given in the proof of Theorem 8 in [2] and a method is demonstrated for any $n$ in [6].

In this study, the solutions of above problems for taxicab regular polygons $P$ in terms of invariants of vectors are investigated. Therefore, an answer is given to the special cases of the open problem posed in 2 .

The study is organized as follows. In Section 2, the conditions of $G$-equivalence of two systems of vectors are given for groups $G=M_{T}(2)$ and $G=D_{4}$. The relations between elements of the complete system of $M_{T}(2)$-invariant functions of two vectors $x_{1}, x_{2}$ is geometrically given. The open problem proposed in 10] is solved for the systems of vectors $\left\{x_{1}, x_{2}, x_{3}\right\}$ and $\left\{y_{1}, y_{2}, y_{3}\right\}$. In Section 3, a minimal complete system of $M_{T}(2)$-invariants functions of the system of vectors $\left\{x_{1}, x_{2}, x_{3}\right\}$ is introduced. In Section 4, both the conditions of the taxicab regularity of Euclidean regular polygons and Euclidean regularity of taxicab regular polygons are given in terms of $M_{T}(2)$-invariants of the vectors. In Section 5, an algorithm to construct taxicab regular polygons and some corresponding examples are given. In Section 6, in addition to the algorithm a procedure is given to the determine the non-existence of taxicab regular $(2 n-1)$-gon having given a line segment as a side for a definite value of $n$, and some corresponding examples are given.

\section{Conditions of G-Equivalence of vectors in taxicab geometry}

Let $G$ be a group.

Definition 1. Two systems of vectors $\left\{u_{1}, u_{2}, u_{3}\right\}$ and $\left\{v_{1}, v_{2}, v_{3}\right\}$ in $R_{T}^{2}$ will be called $G$-equivalent and written by $\left\{u_{1}, u_{2}, u_{3}\right\} \stackrel{G}{\sim}\left\{v_{1}, v_{2}, v_{3}\right\}$ if there exists $F \in G$ such that $v_{j}=F u_{j}$ for all $j=1,2,3$.

Definition 2. A function $f\left(u_{1}, u_{2}, u_{3}\right)$ of vectors $u_{1}, u_{2}, u_{3}$ in $R_{T}^{2}$ will be called $G$-invariant if $f\left(F u_{1}, F u_{2}, F u_{3}\right)=f\left(u_{1}, u_{2}, u_{3}\right)$ for all $F \in G$.

Example 3. Let $u_{1}, v_{1}$ be vectors in $R_{T}^{2}$. Since the group $D_{4}$ is a subgroup of orthogonal group $O(2)$, we have $p\left(u_{1}, v_{1}\right)$ is $D_{4}$-invariant. That is, since $p\left(g u_{1}, g v_{1}\right)=$ $p\left(u_{1}, v_{1}\right)$ for all $g \in D_{4}$, we obtain that the scalar product $p\left(u_{1}, v_{1}\right)$ is $D_{4}$-invariant. Similarly, the function $p\left(u_{1}-v_{1}, u_{1}-v_{1}\right)$ is $M_{T}(2)$-invariant. 
Example 4. Let $u_{1}=\left(u_{11}, u_{12}\right), v_{1}=\left(v_{11}, v_{12}\right)$ be vectors in $R_{T}^{2}$. We define function $q\left(u_{1}, v_{1}\right)=\left(u_{11} u_{12}\right)\left(u_{11} v_{12}+u_{12} v_{11}\right)$. Then $q\left(u_{1}, v_{1}\right)$ is $D_{4}$-invariant. Similarly, the function $q\left(u_{1}-v_{1}, u_{1}-v_{1}\right)$ is $M_{T}(2)$-invariant.

Theorem 5. Let $\left\{u_{1}, u_{2}, u_{3}\right\}$ and $\left\{v_{1}, v_{2}, v_{3}\right\}$ be two systems of vectors in $R_{T}^{2}$. Then following two conditions are equivalent:

(i) $\left\{u_{1}, u_{2}, u_{3}\right\} \stackrel{M_{T}(2)}{\sim}\left\{v_{1}, v_{2}, v_{3}\right\}$

(ii) $\left\{u_{2}-u_{1}, u_{3}-u_{1}\right\} \stackrel{D_{4}}{\sim}\left\{v_{2}-v_{1}, v_{3}-v_{1}\right\}$

Proof. Assume that $\left\{u_{1}, u_{2}, u_{3}\right\} \stackrel{M_{T}(2)}{\sim}\left\{v_{1}, v_{2}, v_{3}\right\}$. Then there exists $F \in M_{T}(2)$ such that $v_{i}=F u_{i}$ for all $i=1,2,3$, where $F$ has the form $F u=g u+b, g \in D_{4}, b \in$ $R_{T}^{2}$. These equalities imply that $v_{i}-v_{1}=g\left(u_{i}-u_{1}\right)$ for all $i=2,3$. This means that $\left\{u_{2}-u_{1}, u_{3}-u_{1}\right\} \stackrel{D_{4}}{\sim}\left\{v_{2}-v_{1}, v_{3}-v_{1}\right\}$.

Conversely, assume that $\left\{u_{2}-u_{1}, u_{3}-u_{1}\right\} \stackrel{D_{4}}{\sim}\left\{v_{2}-v_{1}, v_{3}-v_{1}\right\}$. Then there exists $g \in D_{4}$ such that $v_{i}-v_{1}=g\left(u_{i}-u_{1}\right)$ for all $i=2,3$. Put $b=v_{1}-g u_{1}$. Then $v_{i}=g u_{i}+b$ for all $i=1,2,3$. That is, $\left\{u_{1}, u_{2}, u_{3}\right\} \stackrel{M_{T}(2)}{\sim}\left\{v_{1}, v_{2}, v_{3}\right\}$.

Let $u_{1}, u_{2}, \ldots, u_{m} \in R_{T}^{2}$. We denote the matrix $\left\|p\left(u_{j}, u_{k}\right)\right\|_{j, k=1,2, \ldots, m}$ by $\operatorname{Gr}\left(u_{1}, u_{2}, \ldots, u_{m}\right)$ and its determinant by $\operatorname{det} G r\left(u_{1}, u_{2}, \ldots, u_{m}\right)$.

Below we use the following known proposition (see [5, p.192]).

Proposition 6. Vectors $u_{1}, u_{2}, \ldots, u_{m} \in R_{T}^{2}$ are linearly depended if and only if $\operatorname{det} G r\left(u_{1}, u_{2}, \ldots, u_{m}\right)=0$

Proof. A proof is given [17, p.75].

Example 7. The rank of the system of vectors $X=\left\{x_{1}, x_{2}\right\}$ of vectors in $R_{T}^{2}$ is $D_{4}$-invariant, but it is not $M_{T}(2)$-invariant.

Remark 8. Let $X=\left\{x_{1}, x_{2}\right\}$ and $Y=\left\{y_{1}, y_{2}\right\}$ be two systems of vectors in $R_{T}^{2}$ such that $x_{1} \neq 0$ and $y_{1}=0$. Then the systems $X$ and $Y$ are not $D_{4}$-equivalent. In the case where $x_{1}=y_{1}=0$, the problem of $D_{4}$-equivalence of systems $X$ and $Y$ reduces to the problem of $D_{4}$-equivalence of the systems $\left\{x_{2}\right\}$ and $\left\{y_{2}\right\}$. Therefore we will investigate the problem of $D_{4}$-equivalence of the systems $X$ and $Y$ such that $x_{1} \neq 0$ and $y_{1} \neq 0$.

Theorem 9. Let $X=\left\{x_{1}, x_{2}\right\}$ and $Y=\left\{y_{1}, y_{2}\right\}$ be two systems of vectors in $R_{T}^{2}$ such that $x_{1} \neq 0$ and $y_{1} \neq 0$. Then following two conditions are equivalent:

(i) $\left\{x_{1}, x_{2}\right\} \stackrel{D_{4}}{\sim}\left\{y_{1}, y_{2}\right\}$

(ii) $p\left(x_{i}, x_{j}\right)=p\left(y_{i}, y_{j}\right), q\left(x_{1}, x_{1}\right)=q\left(y_{1}, y_{1}\right)$ and $q\left(x_{1}, x_{2}\right)=q\left(y_{1}, y_{2}\right)$ for all $i=1,2 ; i \leq j$.

Proof. Assume that $\left\{x_{1}, x_{2}\right\} \stackrel{D_{4}}{\sim}\left\{y_{1}, y_{2}\right\}$. Then there exists $g \in D_{4}$ such that $g x_{i}=y_{i}$ for all $i=1,2$. Since the functions $p\left(x_{i}, x_{j}\right), q\left(x_{1}, x_{1}\right)$ and $q\left(x_{1}, x_{2}\right)$ are $D_{4}$-invariants, that is, $p\left(x_{i}, x_{j}\right)=p\left(y_{i}, y_{j}\right), q\left(x_{1}, x_{1}\right)=q\left(y_{1}, y_{1}\right)$ and $q\left(x_{1}, x_{2}\right)=$ 
$q\left(y_{1}, y_{2}\right)$ for all $i=1,2 ; i \leq j$.

Conversely, assume that the conditions $p\left(x_{i}, x_{j}\right)=p\left(y_{i}, y_{j}\right), q\left(x_{1}, x_{1}\right)=q\left(y_{1}, y_{1}\right)$ and $q\left(x_{1}, x_{2}\right)=q\left(y_{1}, y_{2}\right)$ for all $i=1,2 ; i \leq j$ are valid.

Denote by $r(X)$ and $r(Y)$ ranks of the systems $X=\left\{x_{1}, x_{2}\right\}$ and $Y=\left\{y_{1}, y_{2}\right\}$, respectively.

(a) Firstly, consider the case $r(X)=2$. Then there exist vectors $x_{1}, x_{2}$ which are linearly independent. Let $\left\|x_{1} x_{2}\right\|$ be the matrix of column vectors $x_{1}, x_{2}$. Denote by $U$ and $V$ the matrices $\left\|x_{1} x_{2}\right\|$ and $\left\|y_{1} y_{2}\right\|$ and their transpose matrices by $U^{T}$, $V^{T}$, respectively. Let $\operatorname{det} U$ be the determinant of $U$. Linearly independence of $x_{1}, x_{2}$ implies $\operatorname{det} U \neq 0$. $\left\|p\left(x_{i}, x_{j}\right)\right\|_{i, j=1,2}$ is the Gram matrix of vectors $x_{1}, x_{2}$. Then it is easy to see that

$$
U^{T} U=\left\|p\left(x_{i}, x_{j}\right)\right\|_{i, j=1,2}
$$

Since $p\left(x_{i}, x_{j}\right)=p\left(y_{i}, y_{j}\right)$ for all $i, j=1,2$, it is obtained

$$
\left\|p\left(x_{i}, x_{j}\right)\right\|_{i, j=1,2}=\left\|p\left(y_{i}, y_{j}\right)\right\|_{i, j=1,2}
$$

(1) and (2) imply

$$
U^{T} U=V^{T} V
$$

whence

$$
(\operatorname{det} U)^{2}=(\operatorname{det} V)^{2}
$$

Since $\operatorname{det} U \neq 0$, (4) implies that $\operatorname{det} V \neq 0$. That is, the vectors $y_{1}, y_{2}$ are linearly independent. Then there exists a $2 \times 2$-matrix $g$ such that $\operatorname{det} g \neq 0$ and

$$
V=g U
$$

(3) and (5) give the equation

$$
U^{T} U=U^{T} g^{T} g U
$$

Since $\operatorname{det} U \neq 0, \sqrt{6}$ implies $g^{T} g=I$, where $I$ is the identity matrix. This means that $g \in O(2)$. (5) implies $y_{j}=g x_{j}$ for all $j=1,2$. Now we prove that $g \in D_{4}$.

$g \in O(2)$ has the form $g=\left(\begin{array}{cc}a & -b \\ b & a\end{array}\right)$ with $\operatorname{det} g=1$ or $\left(\begin{array}{cc}a & b \\ b & -a\end{array}\right)$ with $\operatorname{det} g=-1$.

Consider the matrix $g=\left(\begin{array}{cc}a & -b \\ b & a\end{array}\right)$ such that $\operatorname{det} g=1$. Let $x_{i}=\left(x_{i 1}, x_{i 2}\right)$ for all $i=1,2$. Since $y_{j}=g x_{j}$ for all $j=1,2$, it is obtained

$$
y_{j}=\left(a x_{j 1}-b x_{j 2}, b x_{j 1}+a x_{j 2}\right)
$$

for all $j=1,2$.

$q\left(x_{1}, x_{1}\right)=q\left(y_{1}, y_{1}\right)$ and $(7)$ imply that

$$
a b=0
$$

(8) and $\operatorname{detg}=a^{2}+b^{2}=1$ give 
(i) If $a=0$, then $b=\mp 1$. So $g=\left(\begin{array}{cc}0 & -1 \\ 1 & 0\end{array}\right)$ or $g=\left(\begin{array}{cc}0 & 1 \\ -1 & 0\end{array}\right)$.

(ii) If $b=0$, then $a=\mp 1$. Therefore $g=\left(\begin{array}{ll}1 & 0 \\ 0 & 1\end{array}\right)$ or $g=\left(\begin{array}{cc}-1 & 0 \\ 0 & -1\end{array}\right)$.

Similarly, consider the matrix $g=\left(\begin{array}{cc}a & b \\ b & -a\end{array}\right)$ such that $\operatorname{det} g=-1$. Let $x_{i}=$ $\left(x_{i 1}, x_{i 2}\right)$ for all $i=1,2$. Since $y_{j}=g x_{j}$ for all $j=1,2$, it is obtained

$$
y_{j}=\left(a x_{j 1}+b x_{j 2}, b x_{j 1}-a x_{j 2}\right)
$$

for all $j=1,2$.

$q\left(x_{1}, x_{1}\right)=q\left(y_{1}, y_{1}\right)$ and $(9)$ imply

$$
a b=0
$$

10 and $\operatorname{det} g=a^{2}+b^{2}=-1$ give

(i) If $a=0$, then $b=\mp 1$. Therefore $g=\left(\begin{array}{ll}0 & 1 \\ 1 & 0\end{array}\right)$ or $g=\left(\begin{array}{cc}0 & -1 \\ -1 & 0\end{array}\right)$.

(ii) If $b=0$, then $a=\mp 1$. Hence $g=\left(\begin{array}{cc}1 & 0 \\ 0 & -1\end{array}\right)$ or $g=\left(\begin{array}{cc}-1 & 0 \\ 0 & 1\end{array}\right)$.

So we obtain that $g \in D_{4}$.

Now, let us prove that there exists $g \in D_{4}$ such that $g x_{i}=y_{i}$ for all $i=$ 1,2. Assume that $g x_{1}=y_{1}, h x_{2}=y_{2}$ such that $g, h \in D_{4}$ and $g \neq h$. Hence the inequality $q\left(x_{1}, x_{2}\right) \neq q\left(y_{1}, y_{2}\right)$ is obtained which a contradiction is to the assumption of the theorem. From the equality $q\left(x_{1}, x_{2}\right)=q\left(y_{1}, y_{2}\right)$, it is obtained that there exists $g \in D_{4}$ such that $g x_{i}=y_{i}$ for all $i=1,2$.

(b) Now, consider the case $r(X)=1$. The conditions of the theorem and Proposition 6 imply that $r(X)=r(Y)$. Let $\tilde{X}$ and $\tilde{Y}$ denote the linear subspaces of $R_{T}^{2}$ spanned by the systems $X=\left\{x_{1}, x_{2}\right\}$ and $Y=\left\{y_{1}, y_{2}\right\}$, respectively. Then $\operatorname{dim}(\tilde{X})=r(X)=r(Y)=\operatorname{dim}(\tilde{Y}) \leq 1$. Since $\operatorname{dim}(\tilde{X})=\operatorname{dim}(\tilde{Y})=1$, there exist vectors $x_{2}$ and $y_{2}$ in $R_{T}^{2}$ such that $p\left(x_{2}, x_{2}\right)=1, p\left(x_{1}, x_{2}\right)=0$ and $p\left(y_{2}, y_{2}\right)=1$, $p\left(y_{1}, y_{2}\right)=0$. Consider the systems $\bar{U}=\left\{x_{1}, x_{2}\right\}$ and $\bar{V}=\left\{y_{1}, y_{2}\right\}$. Then $r(\bar{U})=r(\bar{V})=2$ and $p\left(x_{i}, x_{s}\right)=p\left(y_{i}, y_{s}\right)$ are obtained for all $i, s=1,2$. According to the case $(a)$, there exists $g \in O(2)$ such that $\bar{V}=g \bar{U}$. Similarly, the conditions in the theorem and from the case $(a)$, it is obtained that there exists $g \in D_{4}$ such that $\bar{V}=g \bar{U}$. In particularly, we obtain $y_{j}=g x_{j}$ for all $j=1,2$.

Hence, from $(a)$ and $(b)$, we have $\left\{x_{1}, x_{2}\right\} \stackrel{D_{4}}{\sim}\left\{y_{1}, y_{2}\right\}$.

Corollary 10. According to Theorem 9, the system

$$
\left\{p\left(x_{i}, x_{j}\right), q\left(x_{1}, x_{1}\right), q\left(x_{1}, x_{2}\right), 1 \leq i \leq j \leq 2\right\}
$$

is a complete system of $D_{4}$-invariants of vectors $x_{1}, x_{2}$.

Using the Theorem 5 and Theorem 9 , the following theorem can be obtained. 
Theorem 11. Let $X=\left\{x_{1}, x_{2}, x_{3}\right\}$ and $Y=\left\{y_{1}, y_{2}, y_{3}\right\}$ be two systems of vectors in $R_{T}^{2}$ such that $x_{2}-x_{1} \neq 0$ and $y_{2}-y_{1} \neq 0$. Then following two conditions are equivalent:

(i) $\left\{x_{1}, x_{2}, x_{3}\right\} \stackrel{M_{T}(2)}{\sim}\left\{y_{1}, y_{2}, y_{3}\right\}$

(ii) $p\left(x_{i}-x_{1}, x_{j}-x_{1}\right)=p\left(y_{i}-y_{1}, y_{j}-y_{1}\right), q\left(x_{2}-x_{1}, x_{2}-x_{1}\right)=q\left(y_{2}-y_{1}, y_{2}-y_{1}\right)$ and $q\left(x_{2}-x_{1}, x_{3}-x_{1}\right)=q\left(y_{2}-y_{1}, y_{3}-y_{1}\right)$ for all $i=2,3 ; i \leq j$.

Corollary 12. According to Theorem 11, the system

$$
\left\{p\left(x_{i}-x_{1}, x_{j}-x_{1}\right), q\left(x_{2}-x_{1}, x_{2}-x_{1}\right), q\left(x_{2}-x_{1}, x_{3}-x_{1}\right), 2 \leq i \leq j \leq 3\right\}
$$

is a complete system of $M_{T}(2)$-invariants of vectors $x_{1}, x_{2}, x_{3}$.

Using the Theorem 11, the following theorem gives an answer to the open problem in [10] in terms of $M_{T}(2)$-invariants.

Theorem 13. Let $X=\left\{x_{1}, x_{2}, x_{3}\right\}$ and $Y=\left\{y_{1}, y_{2}, y_{3}\right\}$ be two system of vectors in $R_{T}^{2}$. Then following two conditions are equivalent:

(i) $\left\{x_{1}, x_{2}, x_{3}\right\} \stackrel{M_{T}(2)}{\sim}\left\{y_{1}, y_{2}, y_{3}\right\}$

(ii) $d_{T}\left(x_{i}, x_{j}\right)=d_{T}\left(y_{i}, y_{j}\right)$ and $d_{E}\left(x_{i}, x_{j}\right)=d_{E}\left(y_{i}, y_{j}\right)$ for all $i \neq j$ and $i, j=$ $1,2,3$.

According to Theorem 13 , the system $\left\{d_{T}\left(x_{i}, x_{j}\right), d_{E}\left(x_{i}, x_{j}\right), i, j=1,2,3 ; i \neq j\right\}$ is a complete system of $M_{T}(2)$ - invariants of vectors $x_{1}, x_{2}, x_{3}$.

Specially, the system $\left\{d_{T}\left(x_{1}, x_{2}\right), d_{E}\left(x_{1}, x_{2}\right)\right\}$ is a complete system of $M_{T}(2)$ invariants of vectors $x_{1}, x_{2}$.

Now we investigate relations between elements of the complete system of $M_{T}(2)$ invariant functions of two vectors $x_{1}, x_{2}$

Theorem 14. Let $x_{1}$ be a fixed point in $R_{T}^{2}$. Then for all points $x_{1} \neq x_{2}$, the following statements are hold:

(i) The geometric locus of points $x_{2}$ where $d_{T}\left(x_{1}, x_{2}\right)=d_{E}\left(x_{1}, x_{2}\right)$ are intersection points of taxicab and Euclidean circles with centered $x_{1}$. Geometrically, this is a inscribed quadrilateral.

(ii) The geometric locus of points $x_{2}$ where $d_{T}\left(x_{1}, x_{2}\right)=\sqrt{2} d_{E}\left(x_{1}, x_{2}\right)$ are tangent points of taxicab and Euclidean circles with centered $x_{1}$. Geometrically, this is a circumscribed quadrilateral.

(iii) The geometric locus of points $x_{2}$ where $d_{T}\left(x_{1}, x_{2}\right)<\sqrt{2} d_{E}\left(x_{1}, x_{2}\right)$ are intersection points of taxicab and Euclidean circles with centered $x_{1}$. The number of the points are only eight.

Proof. Let $x_{1}=\left(x_{11}, x_{12}\right)$ and $x_{2}=\left(x_{21}, x_{22}\right)$ be two points in $R_{T}^{2}$ such that $x_{1} \neq x_{2}$. Let $d_{T}\left(x_{1}, x_{2}\right)=a$ and $d_{E}\left(x_{1}, x_{2}\right)=r$, where $a$ and $r$ are positive real numbers. Then, from the equalities $d_{E}\left(x_{1}, x_{2}\right)=\sqrt{p\left(x_{1}-x_{2}, x_{1}-x_{2}\right)}=r$ and $d_{T}\left(x_{1}, x_{2}\right)=\sqrt{p\left(x_{1}-x_{2}, x_{1}-x_{2}\right)+2\left|\left(x_{11}-x_{21}\right)\left(x_{12}-x_{22}\right)\right|}=a$, we have

$$
p\left(x_{1}-x_{2}, x_{1}-x_{2}\right)=r^{2}
$$


and

$$
p\left(x_{1}-x_{2}, x_{1}-x_{2}\right)+2\left|\left(x_{11}-x_{21}\right)\left(x_{12}-x_{22}\right)\right|=a^{2}
$$

The equalities (11) and 12 imply

$$
r^{2}+2\left|\left(x_{11}-x_{21}\right)\left(x_{12}-x_{22}\right)\right|-a^{2}=0
$$

From (13), following three cases are obtained:

(a) if $x_{11}-x_{21}=0$ or $x_{12}-x_{22}=0$, then $r=a$.

Hence the vector $x_{1}-x_{2}$ is parallel to anyone of the lines $x=0$ or $y=0$. See Figure 1 for positions of the vector $x_{1}-x_{2}$.

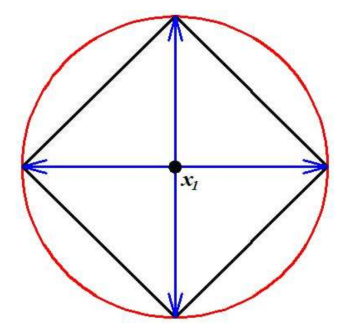

FIGURE 1 . The positions of vectors $x_{2}-x_{1}$ with $d_{T}\left(x_{1}, x_{2}\right)=d_{E}\left(x_{1}, x_{2}\right)$

(b) Let $x_{11}-x_{21} \neq 0$ and $x_{12}-x_{22} \neq 0$. Then there are the following four cases:

(b.1) $x_{11}-x_{21}>0$ and $x_{12}-x_{22}>0$,

(b.2) $x_{11}-x_{21}>0$ and $x_{12}-x_{22}<0$,

(b.3) $x_{11}-x_{21}<0$ and $x_{12}-x_{22}>0$,

(b.4) $x_{11}-x_{21}<0$ and $x_{12}-x_{22}<0$.

(b.1) Let $x_{11}-x_{21}>0$ and $x_{12}-x_{22}>0$. From 13, it is obtained

$$
x_{11}-x_{21}=\frac{a^{2}-r^{2}}{2\left(x_{12}-x_{22}\right)}
$$

Using the equalities (11) and (14), it is obtained

$$
x_{12}-x_{22}=\sqrt{\frac{r^{2} \pm a \sqrt{2 r^{2}-a^{2}}}{2}}
$$

From (15), the following cases are obtained: 
(b.1.1) if $2 r^{2}-a^{2}=0$, then we have $x_{11}-x_{21}=x_{12}-x_{22}=\frac{r}{\sqrt{2}}$. Hence the vector $x_{1}-x_{2}$ is parallel to anyone of the lines $y=x$ or $y=-x$. See Figure 2 for positions of the vector $x_{1}-x_{2}$.

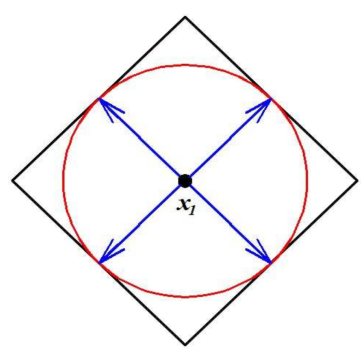

FiguRE 2. The positions of vectors $x_{2}-x_{1}$ with $d_{T}\left(x_{1}, x_{2}\right)=\sqrt{2} d_{E}\left(x_{1}, x_{2}\right)$

(b.1.2) if $2 r^{2}-a^{2}>0$, then we have $x_{11}-x_{21}=x_{12}-x_{22}=\sqrt{\frac{r^{2} \pm a \sqrt{2 r^{2}-a^{2}}}{2}}$.

The cases $(b .2),(b .3)$ and $(b .4)$ are similar to (b.1). Then there exist eight intersection points of taxicab circle and Euclidean circle. See Figure 3 for positions of the vector $x_{1}-x_{2}$.

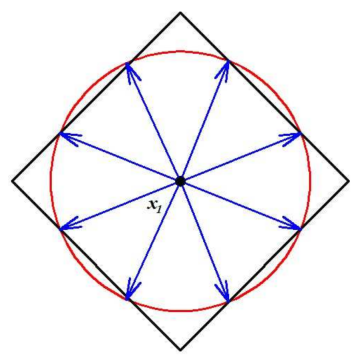

FiguRE 3 . The positions of vectors $x_{2}-x_{1}$ with $d_{T}\left(x_{1}, x_{2}\right)<\sqrt{2} d_{E}\left(x_{1}, x_{2}\right)$

Remark 15. From (iii) in Theorem 14, we have

$$
d_{E}\left(x_{1}, x_{2}\right)<d_{T}\left(x_{1}, x_{2}\right)<\sqrt{2} d_{E}\left(x_{1}, x_{2}\right) .
$$

3. On minimality of the COMPlete System of inVariants of VeCtors

Definition 16. A system $\left\{f_{\tau}, \tau \in Q\right\}$ of $M_{T}(2)$-invariant functions $f_{\tau}\left(x_{1}, x_{2}, x_{3}\right)$ of the systems $\left\{x_{1}, x_{2}, x_{3}\right\}$ in $R_{T}^{2}$ will be called complete if equalities $f_{\tau}\left(x_{1}, x_{2}, x_{3}\right)=$ $f_{\tau}\left(y_{1}, y_{2}, y_{3}\right)$ for all $\tau \in Q$ imply $\left\{x_{1}, x_{2}, x_{3}\right\} \stackrel{M_{T}(2)}{\sim}\left\{y_{1}, y_{2}, y_{3}\right\}$. 
Denote by $K_{i j}(X), L(X)$ and $M(X)$ by the functions $p\left(x_{i}-x_{1}, x_{j}-x_{1}\right)$ for $2 \leq i \leq j \leq 3, q\left(x_{2}-x_{1}, x_{2}-x_{1}\right)$ and $q\left(x_{2}-x_{1}, x_{3}-x_{1}\right)$, respectively.

According to Theorem 11, the system $B=\left\{K_{i j}(X), L(X), M(X), 2 \leq i \leq j \leq 3\right\}$ is a complete system of $M_{T}(2)$-invariant functions of vectors $x_{1}, x_{2}, x_{3}$ in $R_{T}^{2}$.

Definition 17. A complete system $B=\left\{f_{\tau}, \tau \in Q\right\}$ of $M_{T}(2)$-invariant functions $f_{\tau}$ of the system $\left\{x_{1}, x_{2}, x_{3}\right\}$ in $R_{T}^{2}$ will be called minimal if every proper subset of $B$ is not complete.

Theorem 18. The system $B$ is a minimal complete system of $M_{T}(2)$-invariants of vectors $x_{1}, x_{2}, x_{3}$ in $R_{T}^{2}$.

Proof. A proof follows from the following Lemmas 19$] 22$

Lemma 19. The subsystem $B \backslash\left\{K_{23}(X)\right\}$ is not a complete system of $M_{T}(2)$ invariants.

Proof. Consider the following two systems of vectors in $R_{T}^{2}$ :

$X=\left\{x_{1}=(1,2), x_{2}=(3,2), x_{3}=(2,4)\right\}$ and

$Y=\left\{y_{1}=(1,2), y_{2}=(3,2), y_{3}=(3,3)\right\}$. Prove the lemma for $i=2, j=3$. Then we have $K_{22}(X)=K_{22}(Y)=4, K_{33}(X)=K_{33}(Y)=5, L(X)=L(Y)=$ $0, M(X)=M(Y)=0$. Since $K_{23}(X)$ and $K_{23}(Y)$ are $M_{T}(2)$-invariants, $K_{23}(X)=$ $2, K_{23}(Y)=4$, it is obtained that the systems $X$ and $Y$ are not $M_{T}(2)$-equivalent. Hence the subsystem $B \backslash\left\{K_{23}(X)\right\}$ is not complete.

Lemma 20. The subsystem $B \backslash\left\{K_{i i}(X)\right\}$ for any $i=2,3$ is not a complete system of $M_{T}(2)$-invariants.

Proof. Consider the following two systems of vectors in $R_{T}^{2}$ :

$X=\left\{x_{1}=(1,2), x_{2}=(3,2), x_{3}=(4,3)\right\}$ and

$Y=\left\{y_{1}=(1,2), y_{2}=(3,2), y_{3}=(4,4)\right\}$. Prove the lemma for $i=3$. Then we have $K_{22}(X)=K_{22}(Y)=4, K_{23}(X)=K_{23}(Y)=6, L(X)=L(Y)=0, M(X)=$ $M(Y)=0$. Since $K_{33}(X)$ and $K_{33}(Y)$ are $M_{T}(2)$-invariants, $K_{33}(X)=10$, $K_{33}(Y)=13$, it is obtained that the systems $X$ and $Y$ are not $M_{T}(2)$-equivalent. Hence the subsystem $B \backslash\left\{K_{33}(X)\right\}$ is not complete. Similarly, the subsystem $B \backslash\left\{K_{22}(X)\right\}$ is not complete.

Lemma 21. The subsystem $B \backslash\{L(X)\}$ is not a complete system of $M_{T}(2)-$ invariants.

Proof. Consider the following two systems in $R_{T}^{2}$ :

$X=\left\{x_{1}=(1,2), x_{2}=(2,3), x_{3}=(2,1)\right\}$ and

$Y=\left\{y_{1}=(1,2), y_{2}=(1+\sqrt{2}, 2), y_{3}=(1,2-\sqrt{2})\right\}$.

Then we have $K_{22}(X)=K_{22}(Y)=2, K_{23}(X)=K_{23}(Y)=0, K_{33}(X)=K_{33}(Y)=$ $2, M(X)=M(Y)=0$. Since $L(X)$ and $L(Y)$ are $M_{T}(2)$-invariants, $L(X)=2$, $L(Y)=0$, it is obtained that the systems $X$ and $Y$ are not $M_{T}(2)$-equivalent. Hence the subsystem $B \backslash\{L(X)\}$ is not complete. 
Lemma 22. The subsystem $B \backslash\{M(X)\}$ is not a complete system of $M_{T}(2)-$ invariants.

Proof. Consider the following two systems in $R_{T}^{2}$ :

$X=\left\{x_{1}=(1,2), x_{2}=(2,0), x_{3}=(5,4)\right\}$ and

$Y=\left\{y_{1}=(1,2), y_{2}=(2,0), y_{3}=(-3,0)\right\}$.

Then we have $K_{22}(X)=K_{22}(Y)=5, K_{23}(X)=K_{23}(Y)=0, K_{33}(X)=K_{33}(Y)=$ $20, L(X)=L(Y)=4$. Since $M(X)$ and $M(Y)$ are $M_{T}(2)$-invariants, $M(X)=12$, $M(Y)=-12$, it is obtained that the systems $X$ and $Y$ are not $M_{T}(2)$-equivalent. Hence the subsystem $B \backslash\{M(X)\}$ is not complete.

Lemmas 1922 imply that the system $B$ is a minimal complete system of $M_{T}(2)$ invariants. The proof of the theorem is completed.

4. On the Euclidean Regular polygons and taxicab REgular polygons

The following definitions about the taxicab polygons are given in [2, p.27-28] "As in the Euclidean plane, a polygon in the taxicab plane consists of three or more coplanar line segments; the line segments (sides) intersect only at endpoints; each endpoint(vertex) belongs to exactly two line segments; no two line segments with a common endpoint are collinear. If the number of sides of a polygon is $n$ for $n \geq 3$ and $n \in N$, then the polygon is called an $n$-gon. The following definitions for polygons in the taxicab plane are given by means of the taxicab lengths instead of the Euclidean lengths:

Definition 23. A polygon in the plane is said to be taxicab equilateral if the taxicab lengths of its sides are equal.

Definition 24. A polygon in the plane is said to be taxicab equiangular if the measures of its interior angles are equal.

Definition 25. A polygon in the plane is said to be taxicab regular if it is both taxicab equilateral and equiangular.

Definition 24 does not give a new equiangular concept because the taxicab and the Euclidean measure of an angle are the same. That is, every Euclidean equiangular polygon is also the taxicab equiangular, and vice versa. However, since the taxicab plane has a different distance function, Definition 23 and therefore Definition 25 are new concepts."

The following theorem gives us conditions of the taxicab regularity of Euclidean regular polygons in terms of $M_{T}(2)$-invariants, vice versa.

Theorem 26. Let $x_{1}, x_{2}, \ldots, x_{n}$ be vertices of an $n$-sided polygon in the Cartesian plane. Assume that $\left\{x_{i+1}, x_{i}\right\} \stackrel{M_{T}(2)}{\sim}\left\{x_{i+1}, x_{i+2}\right\}$ and the angle between $x_{i}-x_{i+1}$ and $x_{i+2}-x_{i+1}$ has measure $\theta=\frac{\pi(n-2)}{n}$ radian for all $1 \leq i \leq n$. Then the $n$-sided polygon is a taxicab regular $n$-gon and a Euclidean regular $n$-gon, where $n=4$ or $n=8$. 
Proof. For simplicity, let us consider two vertices $x_{1}=(\cos \alpha, \sin \alpha)$ for $\alpha \in(0, \pi / 4)$ and $x_{2}=(0,0)$. Besides, let us start from the vertices. Put $i=1$ and $\left\{x_{2}, x_{1}\right\} \stackrel{M_{T}(2)}{\sim}$ $\left\{x_{2}, x_{3}\right\}$. According to Theorem 5 , we have $\left\{x_{1}-x_{2}\right\} \stackrel{D_{4}}{\sim}\left\{x_{3}-x_{2}\right\}$. Then there exist 8 forms vectors $x_{3}-x_{2}$ such that $x_{3}-x_{2}=(\sin \alpha, \cos \alpha), x_{3}-x_{2}=(-\sin \alpha, \cos \alpha)$, $x_{3}-x_{2}=(-\cos \alpha, \sin \alpha), x_{3}-x_{2}=(-\cos \alpha,-\sin \alpha), x_{3}-x_{2}=(\sin \alpha,-\cos \alpha)$, $x_{3}-x_{2}=(-\sin \alpha,-\cos \alpha)$ and $x_{3}-x_{2}=(-\cos \alpha,-\sin \alpha)$.

(i) Let us consider $x_{3}-x_{2}=(\sin \alpha, \cos \alpha)$. Then the angle between $x_{1}-x_{2}$ and $x_{3}-x_{2}$ has measure $\theta<\pi / 2$. Then $n \leq 3$. Assuming $n \geq 3, n=3$ is obtained . So, $\theta=\pi / 3$ and $\alpha=\pi / 12$.

Now let us consider $\left\{x_{3}, x_{2}\right\} \stackrel{M_{T}(2)}{\sim}\left\{x_{3}, x_{4}\right\}$. Since $n=3$, we obtain that $x_{4}=x_{1}$. Clearly, this is a contradiction. Then $\left\{x_{3}, x_{2}\right\}$ is not $M_{T}(2)$-equivalent to $\left\{x_{3}, x_{4}\right\}$. That is, $n \neq 3$.

(ii) Let us consider $x_{3}-x_{2}=(-\sin \alpha, \cos \alpha)$. Then the angle between $x_{1}-x_{2}$ and $x_{3}-x_{2}$ has measure $\theta=\pi / 2$. Then $n=4$. Since $n=4$, we have $x_{5}=$ $x_{1}$. Let us consider $\left\{x_{3}, x_{2}\right\} \stackrel{M_{T}(2)}{\sim}\left\{x_{3}, x_{4}\right\}$ and $\left\{x_{4}, x_{3}\right\} \stackrel{M_{T}(2)}{\sim}\left\{x_{4}, x_{5}\right\}$. Then the angles between $x_{i}-x_{i+1}$ and $x_{i+2}-x_{i+1}$ for $i=1,2,3$ have measures $\theta=$ $\pi / 2$. Furthermore, we obtain $d_{T}\left(x_{i+1}, x_{i}\right)=d_{T}\left(x_{i+1}, x_{i+2}\right)$ and $d_{E}\left(x_{i+1}, x_{i}\right)=$ $d_{E}\left(x_{i+1}, x_{i+2}\right)$. That is, this is a taxicab square.

(iii) Let us consider $x_{3}-x_{2}=(-\cos \alpha, \sin \alpha)$. Then the angle between $x_{1}-x_{2}$ and $x_{3}-x_{2}$ has measure $\theta=\pi-2 \alpha>\pi / 2$. Then $n>4$. Let us consider $g=\left(\begin{array}{cc}-\cos 2 \alpha & -\sin 2 \alpha \\ \sin 2 \alpha & -\cos 2 \alpha\end{array}\right)$. Since the angle between $x_{2}-x_{3}$ and $x_{4}-x_{3}$ has measure $\theta=\pi-2 \alpha$, we have $g\left(x_{2}-x_{3}\right)=x_{4}-x_{3}$. This implies $x_{4}-x_{3}=(-\cos 3 \alpha, \sin 3 \alpha)$. According to Theorem $5 .\left\{x_{3}, x_{2}\right\} \stackrel{M_{T}(2)}{\sim}\left\{x_{3}, x_{4}\right\}$ implies $\left\{x_{2}-x_{3}\right\} \stackrel{D_{4}}{\sim}\left\{x_{4}-x_{3}\right\}$.

So, $\left\{x_{2}-x_{3}=(\cos \alpha,-\sin \alpha)\right\} \stackrel{D_{4}}{\sim}\left\{x_{4}-x_{3}=(-\cos 3 \alpha, \sin 3 \alpha)\right\}$ is obtained.

From Theorem 11, we have $[(\cos \alpha)(-\sin \alpha)]^{2}=[(-\cos 3 \alpha)(\sin 3 \alpha)]^{2}$. Then this equation implies $\alpha=\pi / 8$. That is, $n=8$. Then the angles between $x_{i}-x_{i+1}$ and $x_{i+2}-x_{i+1}$ for $i=3, \ldots, 7$ have measures $\theta=\pi-2 \alpha$ and $g\left(x_{i}-x_{i+1}\right)=x_{i+2}-x_{i+1}$. This implies $x_{i+2}-x_{i+1}=(-\cos (2 i-1) \alpha, \sin (2 i-1) \alpha)$ and $x_{9}=x_{1}$. This shows that the angles between $x_{i}-x_{i+1}$ and $x_{i+2}-x_{i+1}$ for $i=1,2, \ldots, 7$ have measures $\theta=3 \pi / 4$. Furthermore, we obtain $d_{T}\left(x_{i+1}, x_{i}\right)=d_{T}\left(x_{i+1}, x_{i+2}\right)$ and $d_{E}\left(x_{i+1}, x_{i}\right)=d_{E}\left(x_{i+1}, x_{i+2}\right)$. That is, this is a taxicab regular octagon.

If $\alpha=0$ radians or $\alpha=\pi / 2$ radians, the edges of the polygon are parallel to the lines $x=0$ and $y=0$. The polygon is a taxicab regular square.

If $\alpha=\pi / 4$ radians, the edges of the polygon are parallel to the lines $y=x$ and $y=-x$. The polygon is a taxicab regular square. 
Proofs of the cases $x_{3}-x_{2}=(-\cos \alpha,-\sin \alpha), x_{3}-x_{2}=(\sin \alpha,-\cos \alpha), x_{3}-x_{2}=$ $(-\sin \alpha,-\cos \alpha)$ and $x_{3}-x_{2}=(-\cos \alpha,-\sin \alpha)$ are similar to the proof of $(i),(i i)$ and $($ iii $)$. Hence, we obtain that $n=4$ or $n=8$.

Corollary 27. (1) According to Theorem 26, a taxicab regular octagon is a Euclidean regular iff the slopes of sides of a taxicab regular octagon are equal to $m= \pm \tan (\pi / 8)$ or $m= \pm \tan (3 \pi / 8)$.

(2) According to Theoren 26, a Euclidean regular octagon is a taxicab regular iff the slopes of sides of a Euclidean regular octagon are equal to $m=$ $\pm \tan (\pi / 8)$ or $m= \pm \tan (3 \pi / 8)$.

(3) Every taxicab regular square is also Euclidean regular, vice versa.

Remark 28. ( $i$ ) According to Corollary 27, all taxicab regular octagons are not Euclidean regular, vice versa.

(ii) According to Corollary 27, all taxicab regular squares are Euclidean regular, vice versa.

Corollary 27 and Remark 28 that we derived by using $M_{T}(2)$-invariants, are the same conclusions derived in [2] and [6].

\section{The Proposed Algorithm FOR taxicAB REgular POlygons}

Let $x_{1}$ and $x_{2}$ be vertices of a side of any polygon and $n$ be the number of sides of polygon in the taxicab plane. Consider a side by $\overline{x_{1} x_{2}}$. Since angles in taxicab geometry are measured as in Euclidean geometry, each interior angle of a regular polygon is measured $\theta=\frac{\pi(n-2)}{n}$ radians. Let us introduce the algorithm to construct taxicab regular $n$-gon having $\overline{x_{1} x_{2}}$ as side for a definite value of $n$, with the following steps:

Step 1 The side $\overline{x_{1} x_{2}}$ is rotated through $\beta=\frac{\pi(n+2)}{n}$ radians clockwise about the point $x_{2}$ and is obtained a side $\overline{x_{2} z_{3}}$ such that $z_{3}=x_{2}+g\left(x_{1}-x_{2}\right)$, where $g=\left(\begin{array}{cc}\cos \beta & \sin \beta \\ -\sin \beta & \cos \beta\end{array}\right)$.

Then $d_{E}\left(x_{1}, x_{2}\right)=d_{E}\left(z_{3}, x_{2}\right)$ and the angle between vectors $x_{1}-x_{2}$ and $z_{3}-x_{2}$ are equal to $\theta$.

Step 2 For any point $x_{3}$ on the line passes points $x_{2}$ and $z_{3}$, by solving equations $d_{T}\left(x_{1}, x_{2}\right)=d_{T}\left(x_{3}, x_{2}\right)$ and $p\left(x_{1}-x_{2}, x_{3}-x_{2}\right)=d_{E}\left(x_{1}, x_{2}\right) d_{E}\left(x_{2}, x_{3}\right) \cos \theta$, $x_{3}$ is obtained.

Step 3 Similarly, for all $i=2, \ldots, n-1$, the side $\overline{x_{i} x_{i+1}}$ is rotated through $\beta=$ $\frac{\pi(n+2)}{n}$ radians clockwise about the point $x_{i+1}$ and is obtained a side $\overline{x_{i+1} z_{i+2}}$ such that $z_{i+2}=x_{i+1}+g\left(x_{i}-x_{i+1}\right)$. Then $d_{E}\left(x_{i}, x_{i+1}\right)=d_{E}\left(x_{i+1}, z_{i+2}\right)$ and the angle between vectors $x_{i}-x_{i+1}$ and $z_{i+2}-x_{i+1}$ are equal to $\theta$.

Step 4 For any point $x_{i+2}$ on the line passes points $z_{i+2}$ and $x_{i+1}$, by solving equations $d_{T}\left(x_{i}, x_{i+1}\right)=d_{T}\left(x_{i+2}, x_{i+1}\right)$ and $p\left(x_{i}-x_{i+1}, x_{i+2}-x_{i+1}\right)=$ $d_{E}\left(x_{i}, x_{i+1}\right) d_{E}\left(x_{i+1}, x_{i+2}\right) \cos \theta, x_{i+2}$ is obtained.

Thus, all vertices $x_{3}, x_{4}, \ldots, x_{n+1}$ of the polygon are obtained. 
Step 5 If $x_{n+1}=x_{1}$ and $p\left(x_{n}-x_{1}, x_{2}-x_{1}\right)=d_{E}\left(x_{1}, x_{n}\right) d_{E}\left(x_{1}, x_{2}\right) \cos \theta$, then this is a taxicab regular $n$-gon.

Step 6 If $x_{n+1} \neq x_{1}$, then there is no taxicab regular $n$-gon having $\overline{x_{1} x_{2}}$ as a side.

Remark 29. According to this algorithm, for definite value of $n$, one can constract taxicab regular $2 n$-gons, and determine if there exist $(2 n-1)$-gons, having given a line segment as a side. Clearly, this algorithm is also a tool to give an answer to specal cases of open probleme given in [2].

5.1. Illustrations. In this subsection various examples are given to demonstrate the steps of the proposed algorithm for taxicab regular polygons.

Example 30. Consider a hexagon with vertices $x_{1}=(1,1), x_{2}=(0,0), x_{3}=$ $(-1.57735,0.42265), x_{4}=(-2,2), x_{5}=(-1,3), x_{6}=(0.57735,2.57735)$. This polygon is a taxicab regular hexagon(See Figure 4).

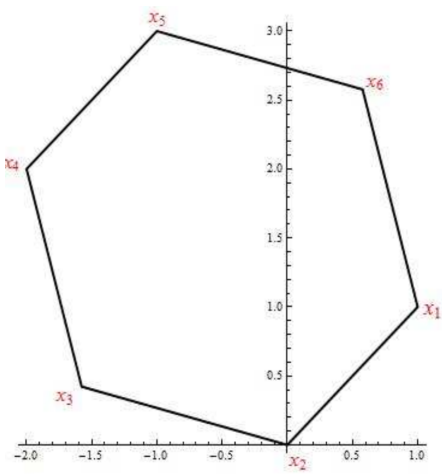

FigurE 4. The taxicab regular hexagon

Example 31. Consider a 10-gon with vertices

$$
\begin{array}{ll}
x_{1}=(2,3), & x_{2}=(1,2), \\
x_{3}=(-0.726543,1.72654), & x_{4}=(-2.05146,2.40162), \\
x_{5}=(-2.72654,3.72654), & x_{6}=(-2.45309,5.45309), \\
x_{7}=(-1.45309,6.45309), & x_{8}=(0.273457,6.72654), \\
x_{9}=(1.59838,6.05146), & x_{10}=(2.27346,4.72654) .
\end{array}
$$

This polygon is a taxicab regular 10-gon.(See Figure 5).

\section{TAXicAB REgularity of POLYGONS With AN ODD NUMBER OF SIDES}

The open problem for $(2 n-1)$-gons posed by 2]: "Does there exist any taxicab regular $(2 n-1)$-gons? "As the given algorithm in Section 5 , the following procedure 


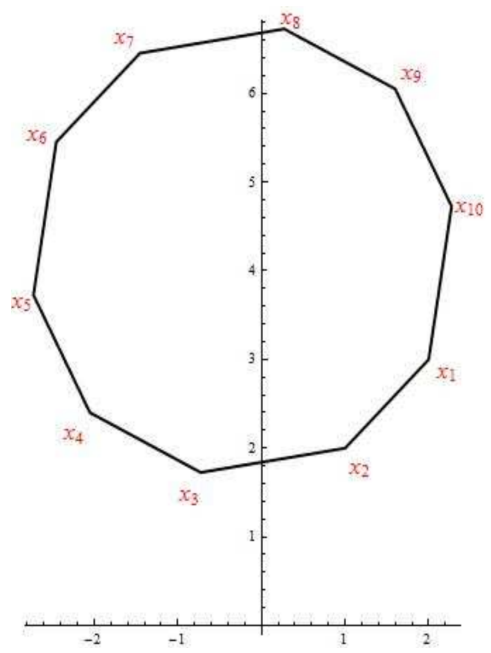

FiguRE 5. The taxicab regular 10-gon

is also a tool to give answer to special cases of open problem given in [2]. That is, for a definite value of $n$, and given a line segment $\overline{x_{1} x_{2}}$, this procedure determines if $(2 n-1)$-gon having $\overline{x_{1} x_{2}}$ as a side exist or not."

Since angles in taxicab geometry are measured as in Euclidean geometry, it is obtained that each interior angle of a regular polygon has measure $\theta=\frac{\pi(n-2)}{n}$ radians.

Let us consider a Euclidean regular $(2 n-1)$-gon with vertices $x_{1}, \ldots, x_{2 n-1}$. For simplicity, let us take a side $\overline{x_{1} x_{2}}$ and denote two vertices by $x_{1}=y_{1}, x_{2}=y_{2}$. Then there exists a point $y_{i+2}$ on the line parallel to the sides $\overline{x_{i+1} x_{i+2}}$ that passes through the point $y_{i+1}$ such that $d_{T}\left(x_{1}, x_{2}\right)=d_{T}\left(y_{i+1}, y_{i+2}\right)$ for each $i=1, \ldots, 2 n-3$. Therefore, the angle between sides $\overline{y_{i} y_{i+1}}$ and $\overline{y_{i+1} y_{i+2}}$ for all $i=1, \ldots, 2 n-3$ equals to $\theta=\frac{\pi(n-2)}{n}$ radians.

But the angle between sides $\overline{y_{2 n-2} y_{2 n-1}}$ and $\overline{y_{2 n-1} y_{1}}$ is not equal to $\theta$, and the inequality $d_{T}\left(y_{1}, y_{2}\right) \neq d_{T}\left(y_{2 n-1}, y_{1}\right)$ holds. If both of these conditions hold at the same time, then $(2 n-1)$-gon with vertices $y_{1}, y_{2}, \ldots, y_{2 n-1}$ is regular, otherwise it is not.

6.1. Illustrations. In this subsection, we give examples related to the procedure introduced above. We have implemented the algoritm proposed in Section 5 in the computer program Mathematica for the examples given in 5.1 Illustrations and 6.1 Illustrations . 
Example 32. Let us consider Euclidean regular triangle with vertices $x_{1}=(2,1), x_{2}=$ $(1,1), x_{3}=(1.5,1.86603)$. Let a side of taxicab regular triangle be $\overline{x_{1} x_{2}}$. Let us denote vertices $x_{1}, x_{2}$ by $y_{1}, y_{2}$,respectively.

Then according to the above procedure, the point $y_{3}=(1.36603,1.63397)$ on the line parallel to the sides $\overline{x_{2} x_{3}}$ that passes through the point $y_{2}$ such that $d_{T}\left(x_{1}, x_{2}\right)=$ $d_{T}\left(y_{2}, y_{3}\right)$ is found. Then the triangle with vertices $y_{1}, y_{2}$ and $y_{3}$ is not taxicab regular. So there is no taxicab regular triangle with the side $\overline{y_{1} y_{2}}$. (See Figure 6 ).

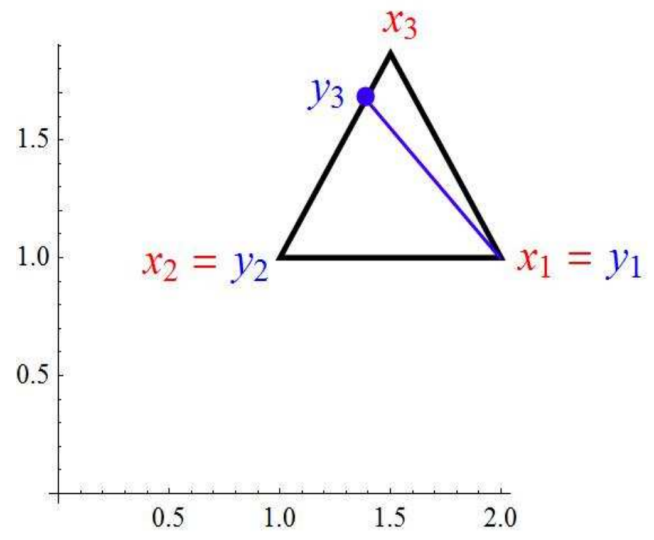

FiguRE 6 . While the triangle with vertices $x_{1}, x_{2}, x_{3}$ is Euclidean regular, the triangle with vertices $y_{1}, y_{2}, y_{3}$ is not taxicab regular.

Example 33. Let us consider Euclidean regular pentagon with vertices

$$
\begin{array}{ll}
x_{1}=(2,1), & x_{2}=(1,1), \\
x_{3}=(0.690983,1.95106), & x_{4}=(1.5,2.53884), \\
x_{5}=(2.30902,1.95106) . &
\end{array}
$$

Let a side of taxicab regular pentagon be $\overline{x_{1} x_{2}}$. Let us denote vertices $x_{1}, x_{2}$ by $y_{1}, y_{2}$,respectively. Then according to the above procedure, the points

$$
\begin{aligned}
& y_{3}=(0.754763,1.75476), \\
& y_{5}=(1.91315,1.75476)
\end{aligned} \quad y_{4}=(1.33395,2.17557),
$$

such that $d_{T}\left(x_{1}, x_{2}\right)=d_{T}\left(y_{2}, y_{3}\right)=d_{T}\left(y_{3}, y_{4}\right)=d_{T}\left(y_{4}, y_{5}\right)$ is found. Clearly, the angle between sides $\overline{y_{4} y_{5}}$ and $\overline{y_{5} y_{1}}$ is not equal to $\theta$, and the inequalty $d_{T}\left(y_{1}, y_{2}\right) \neq$ $d_{T}\left(y_{5}, y_{1}\right)$ holds. The polygon with vertices $x_{1}, x_{2}, x_{3}, x_{4}, x_{5}$ is a Euclidean regular pentagon and but it is not a taxicab regular pentagon with vertices $y_{1}, y_{2}, y_{3}, y_{4}, y_{5}$. (See Figure (7). 


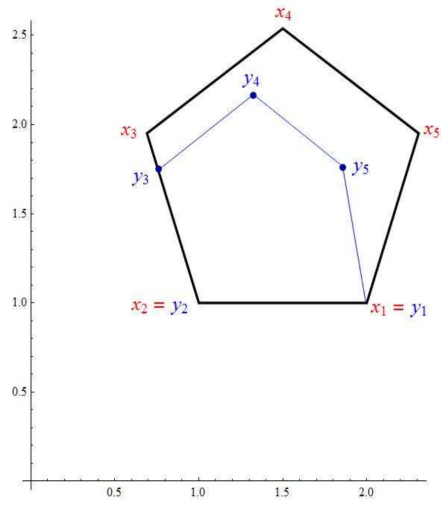

FiguRE 7 . While the pentagon with vertices $x_{1}, x_{2}, x_{3}, x_{4}, x_{5}$ is Euclidean regular,the pentagon with vertices $y_{1}, y_{2}, y_{3}, y_{4}, y_{5}$ is not taxicab regular.

\section{Conclusions}

Finding of an algebraic solution for non-existence of taxicab $(2 n-1)$-gon is difficult. In the special case, choosing an initial side in the algorithm, an algebraic solution can be easily found for $n=3$. Thus, in our paper, the solution of this problem is given numerically. However, the conjecture in [2] still needs to be proven geometrically or algebrically.

Acknowledgements. The authors are very grateful to the reviewer for helpful comments and valuable suggestions.

\section{REFERENCES}

[1] Abrams, P., Some comments on measuring niche overlap, Ecology, 61(1) (1980), 44-49.

[2] Colakoğlu, H. B., Kaya, R., Regular polygons in the taxicab plane, KoG, 12 (2008), 27-33.

[3] Ekici, Kocayusufoğlu, İ., Akça, Z. The norm in taxicab geometry, Turk. J. Math, 22(3) (1998), 295-307.

[4] Goulson, D., Darvill, B., Niche overlap and diet breadth in bumblebees; are rare species more specialized in their choice of flowers?, Apidologie, 35 (2004), 55-63.

[5] Greub, W., Linear algebra, Berlin-Heidelberg-New York:Springer-Verlag, 1967.

[6] Hanson, J. R., Regular polygons in taxicab geometry, Int. J. Math. Educ. Sci. Technol., 45(7) (2014), 1084-1095.

[7] Legendre, P., Gallagher, E., Ecologically meaningful transformations for ordination of species data, Oecologia, 129 (2001), 271-280.

[8] Ören, İ, Çoban, H. A., Some invariant properties of curves in the taxicab geometry, Missouri J. Math. Sci., 26 (2014), 107-114.

[9] Ören,İ., Equivalence conditions of two Bézier Curves in the Euclidean geometry, Iran. J. Technol. A. Sci., 42(3) (2018), 1563-1577.

[10] Schattschneider, D. J., The taxicab group, Am. Math. Mon., 91 (1984), 423-428. 
[11] Slobodchikoff, C. N., Schulz, W. C. Measures of niche overlap, Ecology, 61(7) (1980), 10511055.

[12] Sohn, M. W., Distance and cosine measures of niche overlap, Soc. Networks, 23 (2001), 141-165.

[13] Struble, R. A., Non-Linear differential equations, New York:Mc Graw-Hill, 1962.

[14] Sturmfels, B. Algorithms in invariant theory, Wien:Springer, 2008.

[15] Yüksel, S., Özcan, M., Some regular polygons in the taxicab 3-space, KoG, 19 (2005), 32-41.

[16] Warren, D. L., Glor, R. E., Turelli, M., Environmental niche equivalency versus conservatism: quantitative approaches to niche evolution, Evolution, 62(11) (2008), 2868-2883.

[17] Weyl, H., The classical groups, their invariants and representations, New Delhi: Princeton University Press, 2013.

Current address: İdris ÖREN: Department of Mathematics, Faculty of Science, Karadeniz Technical University, 61080,Trabzon,Turkey.

E-mail address: oren@ktu.edu.tr

ORCID Address: http://orcid.org/0000-0003-2716-3945

Current address: Hüsnü Anıl ÇOBAN (Corresponding Author): Department of Mathematics,

Faculty of Science, Karadeniz Technical University, 61080,Trabzon,Turkey.

E-mail address: hacoban@ktu.edu.tr

ORCID Address: http://orcid.org/0000-0001-8175-4960 